to ESRD was more likely to occur in Texan Hispanics than in other ethnic groups, and in these individuals the time to ESRD was shorter. Young age and, notably, homozygosity for the valine allele of Fc $\gamma$ RIIla, were independently associated with a rapid deterioration of renal involvement, toward ESRD. The investigators found no differences in the distribution of this allele across ethnic groups; hence, the ethnic disparity in the risk of progressive renal involvement in SLE patients remains to be addressed.

Original article Alarcón GS et al. (2006) Time to renal disease and end-stage renal disease in PROFILE: a multiethnic lupus cohort. PLoS Med 3: e396

\section{Good long-term results for surgical treatment of carpal tunnel syndrome}

A Turkish study has shown that open carpal tunnel release (OCTR) surgery is more effective for the long-term treatment of carpal tunnel syndrome (CTS) than splinting or splinting plus local steroid injection. Clinicians generally prefer to use noninvasive treatments for CTS, and despite a lack of consensus on the best treatment for this condition, OCTR is considered to be a final resort. Ucan and colleagues carried out a study to compare the efficacy of splinting, splinting plus local steroids and OCTR as treatments for CTS.

The study included 57 hands diagnosed with mild or moderate idiopathic CTS according to American Association of Electrodiagnostic Medicine guidelines. In total, 23 hands were splinted for 3 months, 23 hands were splinted for 3 months and received a local steroid injection into the carpal tunnel, and 11 hands underwent OCTR.

After 3 months, all groups had improved electrophysiological and clinical outcomes, but splinting plus local steroids was superior in some aspects of symptomatic and functional recovery. Hands in the OCTR group had a greater improvement in median sensory nerve velocities than those in the other two groups. After 6 months, improvements in the OCTR group were sustained, whereas parameters in the other two groups returned to baseline levels.

Despite the potential complications of surgery and the necessarily prolonged period of time away from work that OCTR entails, the authors conclude that OCTR is superior to conservative methods for the long-term treatment of CTS.

Original article Ucan H et al. (2006) Comparison of splinting, splinting plus local steroid injection and open carpal tunnel release outcomes in idiopathic carpal tunnel syndrome. Rheumatol Int 27: 45-51

\section{Adherence to bisphosphonate therapy for osteoporosis}

Nonadherence to bisphosphonate therapy is common in patients with osteoporosis. To investigate the factors associated with such nonadherence in the UK, Carr et al. conducted a telephone-interview survey of 533 women with osteoporosis.

The mean age of participants was 70.7 years; 334 women were taking bisphosphonates at the time of the survey, and 199 had stopped taking bisphosphonates in the previous 12 months. Nonadherence was reported by $52 \%$ of participants overall; $20 \%$ reported occasionally forgetting to take a dose, whereas $13 \%$ occasionally and $26 \%$ regularly decided not to take medication. In addition, $2 \%$ of participants occasionally decided to take more than the prescribed dose. Nonadherence rates were similar for daily and weekly bisphosphonates. Adherence was associated with a previous osteoporotic fracture and prolonged duration of osteoporosis; reasons for nonadherence or nonpersistence were side effects, practical difficulties in taking bisphosphonates, and concerns about addiction or too-frequent dosing. Persistence with therapy was associated with weekly rather than daily bisphosphonates, short disease duration and frequent pain. As study participants were volunteers, and included members of a patient organization who might be more motivated than the general population to comply with their treatment, the authors suggest that rates of adherence and persistence might actually be overestimated in this study.

The authors suggest that patient concerns, particularly about timing of medication and addiction, could be addressed by improved patient literature and the extension of patient choice with the introduction of monthly bisphosphonates.

Original article Carr AJ et al. (2006) Factors associated with adherence and persistence to bisphosphonate therapy in osteoporosis: a cross-sectional survey. Osteoporos Int 17: 1638-1644 